\title{
Studies on stellar rotation ${ }^{\star}$
}

\section{An improvement of the treatment of the brightening distribution of rotating and tidally distorted stars}

\author{
A. Claret ${ }^{\star \star}$ \\ Instituto de Astrofísica de Andalucía, CSIC, Apartado 3004, 18080 Granada, Spain \\ Received 17 March 2003 / Accepted 16 April 2003

\begin{abstract}
We have computed new values of the gravity-darkening exponents $\beta_{1}$ (GDE) incorporating the interior-atmosphere coupling by combining our method developed previously and the properties of modern atmosphere models. The mass range where this coupling is applied is extended in the present work from 0.08 to $40 M_{\odot}$. The effects of the alternative theories of convective transport of energy on GDEs is also investigated. These new values of GDEs are compared to recent observations and we found a good agreement, mainly at the crucial transition phase.

In addition, the gravity-darkening coefficients $y$ were computed for the $u v b$ y $U B V R I J H K$ passbands using recent atmosphere models instead of the black body approximation. All this information is made available in the form of tables and can be obtained from EDP Sciences, or for specific calculations, directly from the author.
\end{abstract}

Key words. stars: fundamental parameters - stars: binaries: close - stars: evolution - stars: rotation

\section{Introduction}

Rotation and/or tides distort the shapes of stars. For a long time, such distortions have been investigated and spherical harmonics used to describe them (see Kopal 1959 for a historical review). In particular, such studies are important in close binary systems where both mechanisms are acting simultaneously: tides tend to elongate the star while rotation tends to flatten it at the poles. The corresponding deviations from a sphere are functions of the rotational rates and of the mass ratio $q$. But, under the physical point of view, not only the "geometrical" changes must be considered. von Zeipel (1924) showed that, for configurations in radiative equilibrium, the emerging flux is not constant on the surface of a distorted star and depends on the value of the local gravity as $T_{\text {eff }}^{4} \propto g^{\beta_{1}}$ where $g$ is the local gravity, $T_{\text {eff }}$ is the effective temperature and $\beta_{1}$ is the gravity-darkening exponent (GDE), a bolometric quantity. Many years later, Lucy (1967) computed GDEs for stars presenting deep convective envelopes. After these works, only two generic values of the gravity-darkening exponent were available: 1 for stars presenting envelopes in radiative equilibrium and 0.32 - as an average - for those where convection is the predominant mechanism of transport of energy.

More recently, (Claret 1998, Paper I) introduced a new method to compute $\beta_{1}$ which embraces both mechanisms of

* Tables 2-26 are only available in electronic form at http://www . edpsciences.org

$\star \star$ e-mail: claret@iaa.es transport of energy simultaneously and the GDEs were presented, for the first time, as continuous functions of the stellar masses, age, chemical composition and mixing-length parameter. Those data were later extended with calculations for very low mass stars (Claret 2000a, Paper II). The covered mass range was 0.08 to $40 M_{\odot}$, which corresponds to effective temperatures between 2000 and $43000 \mathrm{~K}$. In spite of the robustness of the mentioned numerical method, only in the case of the very low mass stars was an appropriated coupling between interior and modern atmosphere models taken into account. In this paper we introduce such an improvement in our stellar evolutionary code in order to investigate its effects on $\beta_{1}$ for stars more massive than $0.6 M_{\odot}$. The influence of adopting alternative treatments of the convective transport of energy is also investigated.

On the other hand, the monochromatic (or band) gravitydarkening coefficients - not to be confused with the gravitydarkening exponent - were usually derived adopting the black body approximation. We introduce here a more realistic approach to the problem, introducing modern atmosphere models instead black body approach.

\section{The effect of the coupling interior-atmosphere on the GDE calculation}

When the components of a binary system are close enough, they are distorted by rotation and tides. This effect has direct consequences on the light curves morphology. The light curves 
changes caused by the tidal/rotation distortions, translated from theoretical point of view, are described by the GDEs. After the pioneering work by von Zeipel (1924), who used a pseudobarotrope, different methods were introduced to compute $\beta_{1}$. Lucy (1967), making use of stellar envelope models, found a mean value of $\beta_{1} \approx 0.32$ for stars in convective equilibrium while Anderson \& Shu (1977) established that the exponent for this kind of star should be zero. This seems to be not confirmed by a systematic semi-empirical derivation of $\beta_{1}$ (Rafert \& Twigg 1980; Pantazis \& Niarchos 1998; Niarchos 2000; Djurasevic et al. 2003). On the other hand, very simple arguments can be used to infer the theoretical value of the GDE: by plotting the evolutionary tracks of 1 and $10 M_{\odot}$ in a $\log g$ versus $\log T_{\text {eff }}$ diagram, and approximating both tracks by straight lines we obtain satisfactory values of $\beta_{1}$ for stars with convective and radiative envelopes, respectively. Although this is a simple and fast method it cannot give us more detailed information and only provides us with mean values of $\beta_{1}$ (see Paper I).

In order to go deeper in to the question we developed a method based on the triangles strategy by Kippenhahn et al. (1967). As the pressure-temperature relationship in an envelope are in practice the same a the stars evolves (provided that it evolves within a very small triangle in the HR diagram) such a property can be expanded to describe an envelope with different temperature distribution, simulating a distorted star with different temperature distribution (see Papers I and II for details). Furthermore, in Paper II it was shown that the GDEs are not only functions of the envelope properties but also of the interior properties since the distortions are described in terms of the apsidal-motion constant, which is a measure of the degree of mass distribution. In addition, the details of the rotational laws are important too. In that paper we introduced the calculations of GDEs for stellar models of very low mass stars for which the coupling interior-atmosphere is indeed a necessary boundary condition. However, for the remaining models the grey approximation was adopted for the atmosphere.

Here we implement such a coupling in our stellar evolution code using modern atmosphere models by Kurucz (2000). The point of contact was selected to be $\log \tau=0$, though we performed several tests up to $\log \tau \approx 2$ which is the maximum optical depth given in the grid of stellar atmosphere models. Such probes were carried out for different stellar masses and evolutionary status. As explained in the previous papers of this series, we selected a $2 M_{\odot}$ model because it is representative since during its evolution it crosses twice the boundary of the radiative-convective zones. The results are presented in Fig. 1 where the continuous line represents the model including the Kurucz models of atmosphere and the dashed line denotes the model using a grey atmosphere. A mixing-length parameter of 1.5 was adopted for both models. The CEFF package was used for the interior solution (Dappen 1994-2000, private communication).

Both models, as expected, are similar at high effective temperature but as the temperature decreases the effects of the greyness are more conspicuous. The transition phase is achieved at different effective temperatures, the non-grey model showing a transition at smaller $T_{\text {eff }}$. The behaviour of the GDEs obtained with the grey simulations are similar to

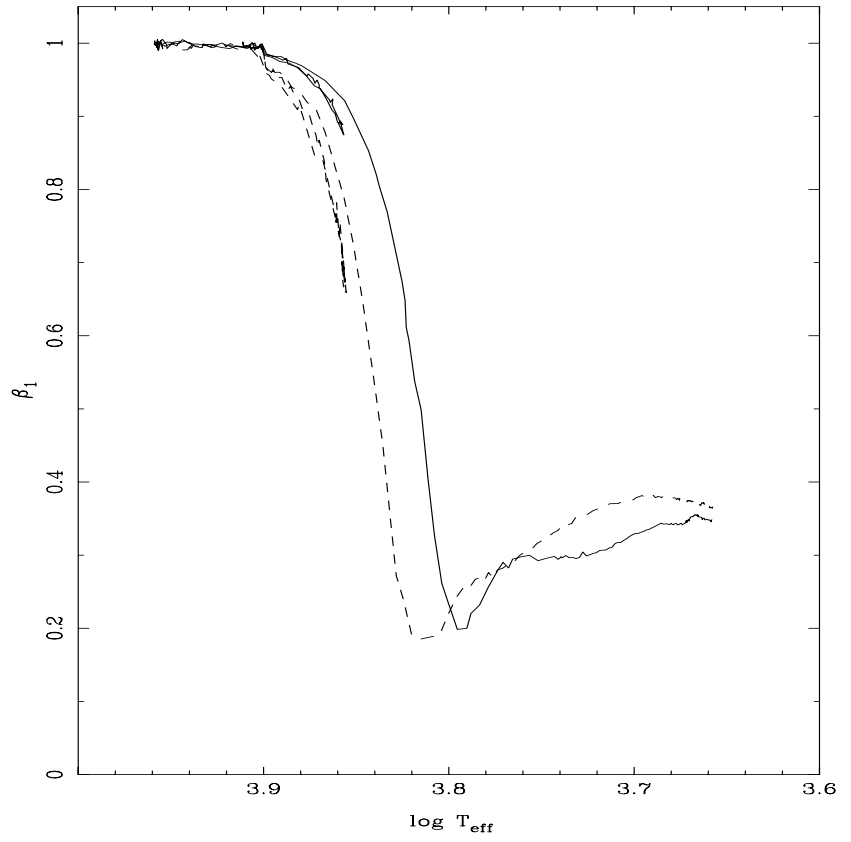

Fig. 1. The theoretical GDE using Kurucz atmosphere models attached to our interior models (continuous line) and those with the grey approximation indicated by the dashed line. Solar chemical composition.

the non-grey ones but displaced to lower effective temperatures and only at lower $T_{\text {eff }}$ are the effects of the switch grey-non grey important. For a given effective temperature (for the more evolved models) the non-grey and grey approximations are different at a given pressure: the temperatures deep inside up to $\log P \approx 4.0$ being systematically higher for the non-grey approximation, the situation is inverted for lower optical depths. The influence of the entropy difference between the envelopes is obvious though not large, as the resulting values of $\beta_{1}$ indicate. The corresponding evolutionary tracks are similar, but for $\log g \approx 3.0$ and a given effective temperature, the non-grey models show slightly larger radii. The values of the GDEs in the grey approximation are systematically larger than its nongrey counterpart for effective temperatures lower than $6300 \mathrm{~K}$.

Another test we can perform concerning the theoretical GDEs calculations is to contrast models based on the mixinglength theory (MLT) and on the Canuto \& Mazzitelli 1991 (CM) approach for the convective transport of energy. For the sake of clarity we compare both options using only the nongrey models. The results are illustrated in Fig. 2. Two features are easily detected in the comparison: 1) the quick increasedecrease of $\beta_{1}$, corresponding to the gravitational contraction just before the hydrogen-shell burning, is more pronounced in the case of MLT; 2) the local minimum of $\beta_{1}$ using MLT occurs at higher effective temperatures than in the CM case; the minimum of $\beta_{1}$ corresponding to $\mathrm{CM}$ is more conspicuous than its counterpart. The reason is linked to the gradient of temperature $\operatorname{dln} T / \mathrm{d} \ln P$ which is small in the MLT approach, mainly in the outermost layers. However, the comparison shown in Fig. 2 should be taken with caution since there is an inconsistency because the attached Kurucz models are always computed using the MLT approach. We have performed a check computing the same models introducing the grey approximation in order 


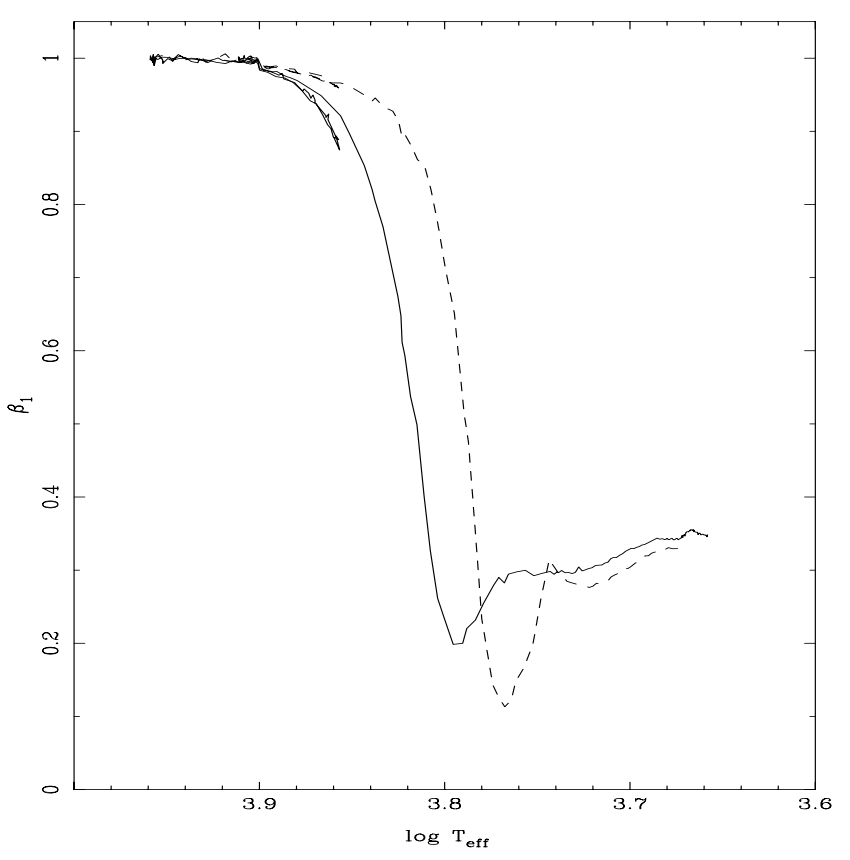

Fig. 2. The theoretical GDE using Kurucz atmosphere models attached to our interior models. The continuous line indicates the models using the mixing-length theory $(\alpha=1.5)$. The dashed line represents the behaviour of the same model but computed adopting the CM theory.

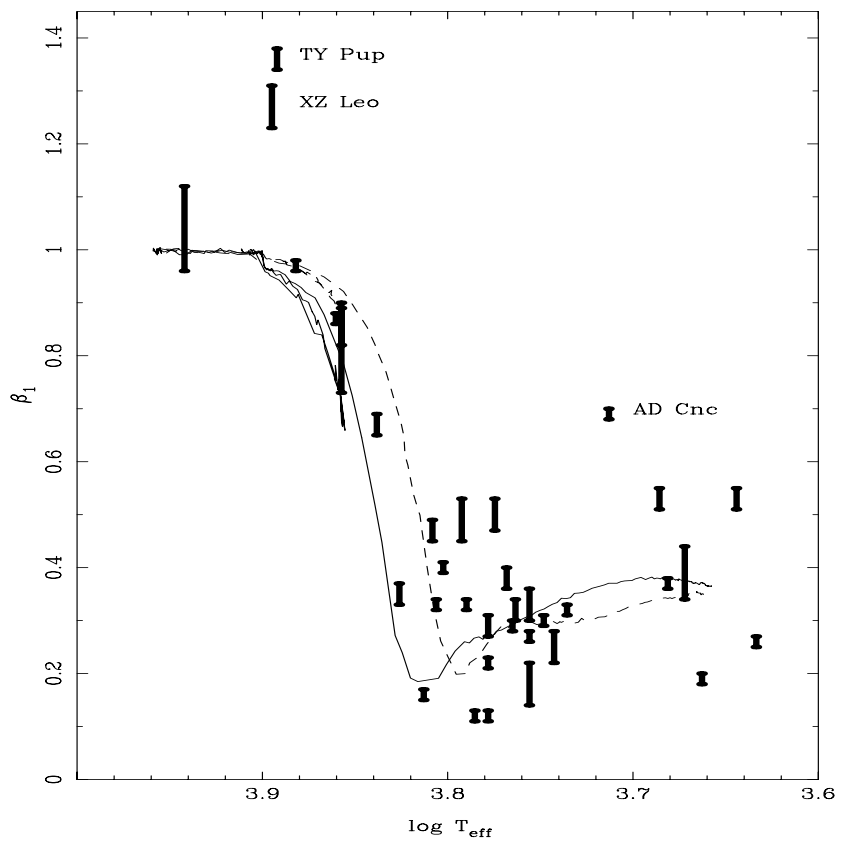

Fig. 3. Comparison between the theoretical GDE and those derived from a recent empirical determination for W Uma-type binaries (Pantazis \& Niarchos 1998; Niarchos 2000). The continuous line denotes the models with grey approximation while the dashed one indicates models using Kurucz atmosphere models.

to eliminate such an inconsistency. Though the results are similar, only a consistent modelization will allows to draw more definitive conclusions.

The calculations of $\beta_{1}$ from the $2 M_{\odot}$ model can also be used to contrast the recent semi-empirical observations with our theoretical predictions. In Fig. 3 we plotted $\beta_{1}$ s computed with Kurucz attached models (dashed line) and those computed using the grey approximation for the atmospheres (continuous line). The observed GDE are from Pantazis \& Niarchos (1998) and Niarchos (2000). The agreement can be considered as good, especially if we take into account that the sample is made up of WW Uma-type binaries which, in general, present strong physical interactions. Only for three systems does the intercomparison yield high $\mathrm{O}-\mathrm{C}$ values. XZ Leo $\left(\beta_{1}=1.27\right)$ and TY Pup $\left(\beta_{1}=1.36\right)$ show a considerable difference with respect to the theoretical value 1.0. In spite of possible problems in the solution of their light curves, the distribution of the angular velocity and other physical complications may be playing some role in these systems, as pointed out by Claret (2000a). The third case, AD Cnc, indicated in the figure refers to a system with both components having envelopes in convective equilibrium. The irradiation should increase the values of $\beta_{1}$ but such an explanation must be considered with caution since other systems of the sample are also being irradiated but they do not present such high $\beta_{1}$ s. An investigation of the effects of the irradiation on the GDE is in preparation (Claret 2003)

\section{The monochromatic gravity-darkening coefficients $y$}

The values of $\beta_{1}$ we just derived - whatever the adopted approximation - refer to bolometric quantities. However, the photometric observations are performed in bands. To connect both entities, Kopal (1959) introduced the concept of the gravitydarkening coefficients, $y$. Such parameters are derived assuming that the distorted configurations radiate like a black body and expanding the ratio between the monochromatic and total radiation in a Taylor series. They are very useful to compute the light distribution of a distorted configuration. Of course, the black body radiation is not a good approximation and more elaborate models are need to permit a more complete light curve analysis. Fortunately, modern models of stellar atmospheres are now available computed with a wide variety of input physics. Using the same grid of stellar atmosphere models by Kurucz adopted in the previous section, we derive the $y$ s for the most widely used photometric systems, say, $u v b$ y $U B V R I J H K$. For $2000 \mathrm{~K} \leq T_{\mathrm{eff}} \leq 9800 \mathrm{~K}$ a grid of PHOENIX models are also used (Hauschildt 2000). We adopt for $y$ the following approximation (Martynov 1973):

$y\left(\lambda, T_{\text {eff }}, Z, \log g, v\right)=\frac{1}{4}\left(\frac{\mathrm{d} \ln I}{\mathrm{~d} \ln T}\right)_{g}$

where $\lambda$ denotes the wavelength, $Z$ the metal content, $v$ the microturbulent velocity, $I$ is the intensity at a given wavelength and the subscript $g$ indicates that the derivatives are calculated at a constant surface gravity. The results can be seen in Fig. 4 for the $u v$ b y $U B V R I J H K$ photometric systems. In general the black body approximation cannot be considered as good, except for very small intervals of effective temperatures. For a given filter, the differences may be large, especially for passbands with short effective wavelength. A common characteristic of all passbands is that at $\log T_{\text {eff }} \approx 3.9$ a jump in the 
A. Claret: Studies on stellar rotation. III.
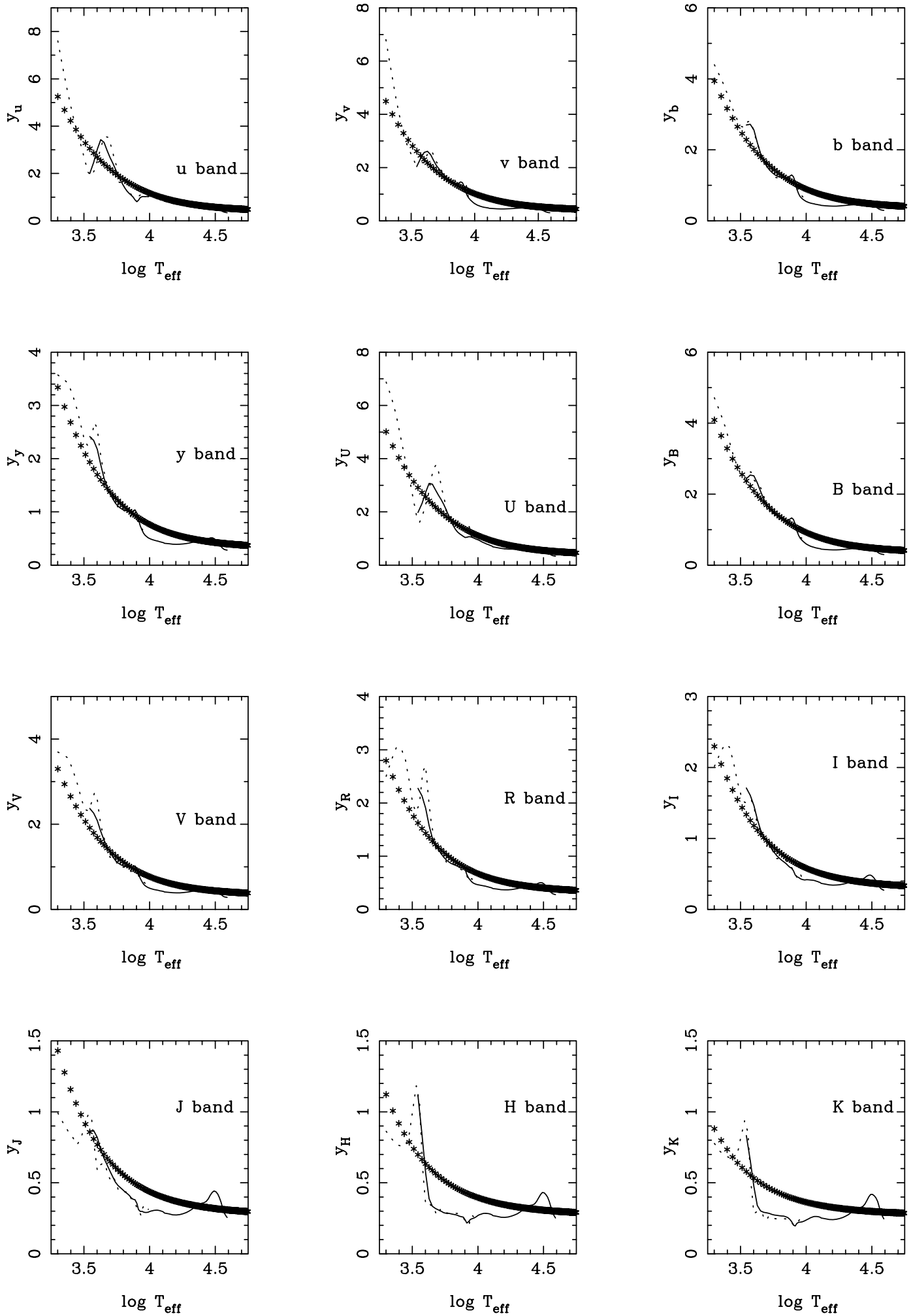

Fig. 4. The band gravity-darkening coefficients $y$ as a function of the effective temperature and photometric passbands. The asterisks represent the back body approach and the continuous line represents ATLAS models with $\log g=4.0, v=2 \mathrm{~km} \mathrm{~s}^{-1}, \log [\mathrm{M} / \mathrm{H}]=0$. The dotted $\operatorname{line}$ represents PHOENIX models for the same input parameters as before.

values of $y$ is present and possibly this is connected to the onset of convection. This was already detected in the calculation of the limb-darkening coefficients (Díaz-Cordobés et al. 1995; and Claret 2000b, for example). The differences between the ys computed using ATLAS (continuous lines) and those from the PHOENIX code (dotted lines) are not so large, for fixed input parameters. 
Table 1. Gravity-darkening exponents (bolometric) and gravity-darkening coefficients for $u v b$ y $U B V R I J H K$.

\begin{tabular}{|c|c|c|c|c|c|c|}
\hline Name & Source & range $T_{\text {eff }}$ & range $\log g$ & $\log [\mathrm{M} / \mathrm{H}]$ & Vel turb. & $y$ \\
\hline Table 2 & ATLAS & $3500 \mathrm{~K}-50000 \mathrm{~K}$ & $0.0-5.0$ & -5.0 & $2 \mathrm{~km} \mathrm{~s}^{-1}$ & 12 filters \\
\hline Table 3 & ATLAS & $3500 \mathrm{~K}-50000 \mathrm{~K}$ & $0.0-5.0$ & -4.5 & $2 \mathrm{~km} \mathrm{~s}^{-1}$ & 12 filters \\
\hline Table 4 & ATLAS & $3500 \mathrm{~K}-50000 \mathrm{~K}$ & $0.0-5.0$ & -4.0 & $2 \mathrm{~km} \mathrm{~s}^{-1}$ & 12 filters \\
\hline Table 5 & ATLAS & $3500 \mathrm{~K}-50000 \mathrm{~K}$ & $0.0-5.0$ & -3.5 & $2 \mathrm{~km} \mathrm{~s}^{-1}$ & 12 filters \\
\hline Table 6 & ATLAS & $3500 \mathrm{~K}-50000 \mathrm{~K}$ & $0.0-5.0$ & -3.0 & $2 \mathrm{~km} \mathrm{~s}^{-1}$ & 12 filters \\
\hline Table 7 & ATLAS & $3500 \mathrm{~K}-50000 \mathrm{~K}$ & $0.0-5.0$ & -2.5 & $2 \mathrm{~km} \mathrm{~s}^{-1}$ & 12 filters \\
\hline Table 8 & ATLAS & $3500 \mathrm{~K}-50000 \mathrm{~K}$ & $0.0-5.0$ & -2.0 & $2 \mathrm{~km} \mathrm{~s}^{-1}$ & 12 filters \\
\hline Table 9 & ATLAS & $3500 \mathrm{~K}-47500 \mathrm{~K}$ & $0.0-5.0$ & -1.5 & $2 \mathrm{~km} \mathrm{~s}^{-1}$ & 12 filters \\
\hline Table 10 & ATLAS & $3500 \mathrm{~K}-50000 \mathrm{~K}$ & $0.0-5.0$ & -1.0 & $2 \mathrm{~km} \mathrm{~s}^{-1}$ & 12 filters \\
\hline Table 11 & ATLAS & $3500 \mathrm{~K}-50000 \mathrm{~K}$ & $0.0-5.0$ & -0.5 & $2 \mathrm{~km} \mathrm{~s}^{-1}$ & 12 filters \\
\hline Table 12 & ATLAS & $3500 \mathrm{~K}-50000 \mathrm{~K}$ & $0.0-5.0$ & -0.3 & $2 \mathrm{~km} \mathrm{~s}^{-1}$ & 12 filters \\
\hline Table 13 & ATLAS & $3500 \mathrm{~K}-50000 \mathrm{~K}$ & $0.0-5.0$ & -0.2 & $2 \mathrm{~km} \mathrm{~s}^{-1}$ & 12 filters \\
\hline Table 14 & ATLAS & $3500 \mathrm{~K}-50000 \mathrm{~K}$ & $0.0-5.0$ & -0.1 & $2 \mathrm{~km} \mathrm{~s}^{-1}$ & 12 filters \\
\hline Table 15 & ATLAS & $3500 \mathrm{~K}-50000 \mathrm{~K}$ & $0.0-5.0$ & 0.0 & $2 \mathrm{~km} \mathrm{~s}^{-1}$ & 12 filters \\
\hline Table 16 & ATLAS & $3500 \mathrm{~K}-50000 \mathrm{~K}$ & $0.0-5.0$ & +0.1 & $2 \mathrm{~km} \mathrm{~s}^{-1}$ & 12 filters \\
\hline Table 17 & ATLAS & $3500 \mathrm{~K}-50000 \mathrm{~K}$ & $0.0-5.0$ & +0.2 & $2 \mathrm{~km} \mathrm{~s}^{-1}$ & 12 filters \\
\hline Table 18 & ATLAS & $3500 \mathrm{~K}-50000 \mathrm{~K}$ & $0.0-5.0$ & +0.3 & $2 \mathrm{~km} \mathrm{~s}^{-1}$ & 12 filters \\
\hline Table 19 & ATLAS & $3500 \mathrm{~K}-45000 \mathrm{~K}$ & $0.0-5.0$ & +0.5 & $2 \mathrm{~km} \mathrm{~s}^{-1}$ & 12 filters \\
\hline Table 20 & ATLAS & $3500 \mathrm{~K}-40000 \mathrm{~K}$ & $0.0-5.0$ & +1.0 & $2 \mathrm{~km} \mathrm{~s}^{-1}$ & 12 filters \\
\hline Table 21 & ATLAS & $3500 \mathrm{~K}-50000 \mathrm{~K}$ & $0.0-5.0$ & 0.0 & $0 \mathrm{~km} \mathrm{~s}^{-1}$ & 12 filters \\
\hline Table 22 & ATLAS & $3500 \mathrm{~K}-50000 \mathrm{~K}$ & $0.0-5.0$ & 0.0 & $1 \mathrm{~km} \mathrm{~s}^{-1}$ & 12 filters \\
\hline Table 23 & ATLAS & $3500 \mathrm{~K}-50000 \mathrm{~K}$ & $0.0-5.0$ & 0.0 & $4 \mathrm{~km} \mathrm{~s}^{-1}$ & 12 filters \\
\hline Table 24 & ATLAS & $3500 \mathrm{~K}-50000 \mathrm{~K}$ & $0.0-5.0$ & 0.0 & $8 \mathrm{~km} \mathrm{~s}^{-1}$ & 12 filters \\
\hline Table 25 & PHOENIX & $2000 \mathrm{~K}-9800 \mathrm{~K}$ & $3.5-5.0$ & 0 & $2 \mathrm{~km} \mathrm{~s}^{-1}$ & 12 filters \\
\hline Table 26 & $\beta_{1}$ for ZAMS models (Paper II) & & & & & \\
\hline
\end{tabular}

As Eq. (1) indicates, $y$ is also a function of other input parameters of the atmosphere models, say, metallicity, surface gravity and microturbulent velocity. The dependence of $y$ on the metallicity is more obvious for the $u, U, H$ and $K$, that is, for the extreme values of our set of passbands, the larger effects being detected for low effective temperatures.

The influence of the surface gravity is not so important, though it is clearly detected. Except for the lower effective temperature, the differences between models with $\log =5$ and $\log g=2.5$ are very small. Concerning the microturbulent velocity, the differences between models with $v=2$ and $8 \mathrm{~km} \mathrm{~s}^{-1}$ are only detectable for lower effective temperatures and for the passbands $u, b, U$ and $B$, as expected.

\section{Conclusions and final remarks}

New values of GDEs, taking into account the coupling interioratmosphere, were obtained by combining our method developed in Paper I and the properties of modern atmosphere models. In this way, the mass range where this coupling is applied was extended from 0.08 up to $40 M_{\odot}$. Furthermore, alternative theories of convective transport of energy were also checked. A model of $2 M_{\odot}$, which "mimics" during its evolution models with convective and radiative envelopes, was used a standard to compare our theoretical results with the more recent semiempirical determination of $\beta_{1}$ provided by Pantazis \& Niarchos (1998) and Niarchos (2000). These observed GDE are more accurate than those by Rafert \& Twigg (1980) and provide a better definition of the transition region. The comparison shows a good agreement. However, it is not possible yet to discriminate, for example, between the currently adopted theories of convection, or between grey and no-grey approach (though in this case, the implementation of a more elaborate model of the atmosphere certainly works better than the grey approximation).

On the other hand, new values of the gravity-darkening coefficients $y$ are computed using modern models of stellar atmosphere for 23 grids covering a $\log g$ range between 0.0 and 5.0, $-5.0 \leq \log [\mathrm{M} / \mathrm{H}] \leq+1,2000 \mathrm{~K} \leq T_{\text {eff }} \leq 50000$ and values of the microturbulent velocities between 0 and $8 \mathrm{~km} \mathrm{~s}^{-1}$. These grids supersede the old values of $y$, often based on the black body approximation. Table 1 summarises the content of the 24 tables which are available electronically. The implementation of the tables of $\beta_{1}$ and $y \mathrm{~s}$ in light curves and/or transit of extrasolar planets codes is straightforward. We are able to provide tables for each case to interested readers. As a final comment and in order to make the results for low mass stars available, we give in Table 26 the values of $\beta_{1}$ from Paper II since in that paper they were shown only in figure format. 
Acknowledgements. Jens Viggo Clausen is acknowledged for many fruitful discussions. P. G. Niarchos is also acknowledged for stimulating correspondence. I am grateful to the referee for helpful comments. The Spanish DGYCIT (PB98-0499) is gratefully acknowledged for its support during the development of this work.

\section{References}

Anderson, L., \& Shu, F. H. 1977, ApJ, 214, 798

Canuto, V. M., \& Mazzitelli, I. 1991, ApJ, 370, 295

Claret, A. 1998, A\&AS, 131, 395 (Paper I)

Claret, A. 2000a, A\&A, 359, 289 (Paper II)

Claret, A. 2000, A\&A, 363, 1081

Dappen, W. 1994-2000, private communication
Díaz-Cordovés, J., Claret, A., \& Giménez, A. 1995, A\&AS, 110, 329

Djurasevic, G., Rovithis-Livaniou, H., Rovithis, P., Georgiades, N., \& Erkapic, S. 2003, A\&A, 402, 667

Hauschildt, P. H. 2000, private communication

Lucy, L. B. 1967, Z. Astrophys., 65, 89

Kopal, Z. 1959, Close Binary Systems (Chapman \& Hall)

Kurucz, R. L. 2000, private communication

Niarchos, P. G. 2000, in Variable Stars as Essential Astrophysical Tools, ed. C. Ibanoglu, 631

Martynov, D. Ya. 1973, in Eclipsing Variable Stars, 128, ed. V. P. Tsesevich (IPST Astrophysics Library)

Pantazis, G., \& Niarchos, P. G. 1998, A\&A, 335, 199

Rafert, J. B., \& Twigg, L. W. 1980, MNRAS, 193, 79

von Zeipel, H. 1924, MNRAS, 84, 665 